\title{
Utilizando Acelerômetros de Smartphones para Prevenção de Acidentes de Trânsito
}

\author{
M. Mendelson* A. Zaghetto ${ }^{* *}$ C. Zaghetto ${ }^{* * *}$ A. Costa ${ }^{* * *}$ \\ F. Vidal ${ }^{* *}$ \\ * Departamento de Engenharia de Software, Universidade de Brasília, \\ DF. \\ ** Departamento de Ciência da Computação, Universidade de Brasília, \\ DF. \\ *** Escola de Tecnologia, Centro Universitário Projeção, DF.
}

\begin{abstract}
This paper presents a method based on Artificial Neural Networks that uses signals captured from conventional cell phone accelerometers to identify user's displacement patterns. The patterns are classified according to the location of the device (pocket, hand or console) and the way the user moves (using a car or walking). Such method enables the identification of risky situations that can lead to accidents, such as driving or walking using a smartphone. Tests show that the proposed method is able to identify one of 10 possible transport modes with an average hit rate of $95 \%$.

Resumo: Este artigo apresenta um método baseado em Redes Neurais Artificiais que, utilizando sinais capturados a partir de acelerômetros de telefones celulares convencionais, é capaz de identificar padrões de deslocamento do usuário definidos pela localização do dispositivo (no bolso, na mão ou no console) e pela forma como o usuário se desloca (utilizando um carro ou a pé). Tal método possibilita a identificação de situações de risco que podem culminar em acidentes, como dirigir ou andar utilizando um smartphone. Os testes mostram que o método proposto é capaz de identificar uma dentre 10 possíveis classes de transporte com uma taxa de acerto médio de $95 \%$.
\end{abstract}

Keywords: Accident Prevention; Accelerometers; Artificial Neural Networks; Smartphone; Signal Processing.

Palavras-chaves: Prevenção de Acidentes; Acelerômetros; Redes Neurais Artificiais; Smartphone; Processamento de Sinais.

\section{INTRODUÇÃO}

Há algum tempo, telefones celulares deixaram de ser apenas dispositivos de telecomunicação verbal. Na verdade, tais dispositivos evoluíram para os chamados smartphones e tornaram-se computadores portáteis com considerável capacidade de processamento e memória. Estes dispositivos, além de serem pequenos computadores, possuem diversos sensores embarcados, dentre os quais destacam-se os acelerômetros, que são capazes de estimar a aceleração do telefone em todas as direções no espaço tridimensional, conforme ilustrado na Figura 2. Entretanto, o uso destes dispositivos ao dirigir e andar na rua tem se tornado cada vez mais comum, o que oferece grande risco de acidentes. Ao analisar 6 capitais europeias, constatou-se que cerca de $17 \%$ dos pedestres utilizam seus smartphones enquanto andam na rua (Sigloch, 2016). Em outro estudo, ao minerar dados no Twitter, vê-se que $18 \%$ dos tweets indicam que o usuário estava jogando e dirigindo ao mesmo tempo (Ayers et al., 2016).

No presente artigo será apresentado um método para se detectar padrões de deslocamento a partir do acelerômetro de um telefone celular, possibilitando, assim, identificar situações de risco de acidentes.
Na seção 2, serão apresentados os trabalhos correlatos. Em seguida, na seção 3, o método proposto é apresentado e, nas seções 4 e 5 , são analisados os resultados e apresentadas as conclusões, respectivamente.

\section{TRABALHOS CORRELATOS}

A utilização de smartphones como ferramentas de predição das situações em que seus portadores se encontram vem sendo cada vez mais explorada. Em Shapsough et al. (2016) os autores propõem o reconhecimento de emoções utilizando o padrão de uso do teclado.

Aplicações em e-Health que, como o presente trabalho também fazem uso de acelerômetros, estão igualmente se tornando comuns. Dentre elas, há abordagens de rastreadores de atividades físicas em exercícios (Anjum and Ilyas, 2013; Kwapisz et al., 2011; Aguiar et al., 2014; Figueira et al., 2016), que buscam identificar o tipo de atividade física que um usuário está praticando.

Em Anjum and Ilyas (2013), foi realizado um estudo sobre o rastreamento de atividades físicas para que o usuário tenha um estilo de vida mais saudável e, para tal, é realizado o cálculo de seu gasto calórico. Para que os dados pudessem ser coletados, foram utilizados acelerômetro, 
giroscópio e GPS, com uma taxa de aquisição de dados de frequência de $15 \mathrm{~Hz}$. Esse método monta janelas de 5 segundos de dados e extrai diversas características, tais como média, desvio padrão, magnitude da aceleração, energia espectral, entropia no domínio da frequência etc. A classificação é realizada por meio de Árvore de Decisão, KNN, Naïve Bayes e SVM.

O trabalho apresentado em Kwapisz et al. (2011) se interessa pela determinação dos hábitos do usuário. O único sensor utilizado nas aquisições é o acelerômetro do próprio smartphone, que foi configurado para funcionar em uma frequência de $20 \mathrm{~Hz}$. O método realiza a classificação utilizando janelas de 10 segundos de dados. Para cada janela, são extraídas 43 características, que são apresentadas a 3 classificadores: Árvore de Decisão J48, Regressão Logística e Redes Neurais Artificiais Multicamada. As Redes Neurais Artificiais Multicamada foram o classificador que apresentou a maior taxa de acerto média.

Por sua vez, em Aguiar et al. (2014) o foco é detectar posturas de inatividade e de atividades físicas, incluindo o cálculo do gasto energético do usuário. Os dados são adquiridos através de um acelerômetro funcionando a $33,33 \mathrm{~Hz}$ e são divididos em janelas de 5 segundos. A partir dos 3 eixos do acelerômetro, foram derivadas diversas componentes do sinal, tais como: magnitude da aceleração, ângulos entre o vetor aceleração e cada um dos eixos do celular e diversas métricas no domínio da frequência. No total, são extraídas 28 componentes do sinal. De cada componente, são extraídas 12 características: média, mediana, máximo, mínimo, raiz quadrática média, desvio padrão, desvio médio, intervalo intercuartil, energia, entropia, skewness e kurtosis. No total, cada janela de 5 segundos fornece 336 características, que são apresentadas a uma Árvore de Decisão.

Algumas aplicações, ainda, focam na detecção do modo de transporte para que o planejamento de empresas e órgãos possam realizar melhor seus planejamentos. Em Widhalm et al. (2012), foram utilizados um acelerômetro e o GPS. Quando o GPS não estava disponível, a rede do celular foi utilizada para calcular a posição aproximada do dispositivo. Os tamanhos das janelas de dados são definidos dinamicamente em tempo de execução, variando em momentos em que o usuário está em estado estacionário, movendo-se lentamente ou movendo-se rapidamente. Para isso, são definidos limiares que caracterizam cada uma dessas situações. O tamanho máximo da janela, entretanto, é definido em 2 minutos. O classificador utilizado é o Modelo Oculto de Markov Discreto.

Outra abordagem em e-Health é a estimação do risco que uma atividade fora de casa representa para uma pessoa idosa (Mejia et al., 2016; Cardoso et al., 2016).

Em Cardoso et al. (2016), foi utilizada uma técnica que coleta dados a partir de um acelerômetro operando a uma frequência de $33,33 \mathrm{~Hz}$ e de um Wi-Fi. Os dados são organizados em janelas de 5 segundos. A partir do sinal original ( $x, y, z$, magnitude), são derivadas $21 \mathrm{com}-$ ponentes do sinal. Para cada componente, são extraídas 17 características, sendo elas: média, mediana, máximo, mínimo, raiz quadrática média, desvio padrão, desvio médio, intervalo intercuartil, média mínima e média máxima, altura de pico, altura média de pico, contagem cruzada média, entropia, energia, skewness e kurtosis. Sendo assim, cada janela de 5 segundos resulta em 357 características, que são apresentadas a 3 classificadores: Árvore de Decisão J48, SMO e Naïve Bayes.

O trabalho em Mejia et al. (2016), por sua vez, utiliza GPS para identificar se o idoso está realizando alguma atividade fora do comum e, assim, calcular o risco de acidentes do idoso naquela situação.

Ainda em aplicações de e-Health, há a proposta de uma ferramenta para avaliação dos sintomas da doença de Parkinson (Sahyoun et al., 2016).

Também há aplicações focadas na evolução de pacientes idosos em fisioterapia. Em Figueira et al. (2016), foram utilizados um acelerômetro, com taxa de aquisição de 30 $\mathrm{Hz}$, e um barômetro, com taxa de aquisição de $5 \mathrm{~Hz}$. Os dados são separados em janelas de 5 segundos e, então, são extraídas 29 características de cada janela. Após a extração das características, elas são normalizadas. Finalmente, elas são apresentadas a uma Árvore de Decisão.

Por fim, há aplicações que visam a detecção do modo de transporte em geral. Nessas aplicações, procura-se identificar se um indivíduo está se locomovendo em um carro, ônibus, bicicleta, bonde, trem, metrô, caminhada, moto, a pé ou se encontra-se em estado estacionário. Tais classificações são realizadas por meio do uso de técnicas como Naïve Bayes, árvores de decisão, redes neurais convolucionais, máquina de vetores de suporte, regressão logística, perceptron multicamada e modelo oculto de Markov. O método aqui proposto utiliza o acelerômetro para a obtenção dos dados e redes neurais artificiais como classificador.

A Tabela 1 apresenta um compilado dos trabalhos aqui mencionados referentes a detecção do modo de transporte.

\section{MÉTODO PROPOSTO}

Considerando que o objetivo do presente trabalho é detectar, com o auxílio de um acelerômetro e fazendo uso de um classificador, se o smartphone está sendo transportado de forma a gerar riscos de acidentes, uma base de dados foi criada para treinar e testar uma rede neural artificial do tipo feedforward multicamadas. Aqui destacase que o classificador proposto utiliza apenas sinais de um acelerômetro para realizar a classificação, portanto não depende de conexões de dados, GPS ou outros. A base de dados conta com 10 possíveis classes $\tau$, resumidas no diagrama apresentado na Figura 1. O celular pode estar na posse de alguém que está dentro de um carro $\left(a_{1}\right)$ ou não $\left(a_{2}\right)$. Pode-se observar se o usuário está transportando o smartphone no console do carro $\left(a_{1} b_{1}\right)$, em mãos $\left(a_{1} b_{2}\right.$ e $\left.a_{2} b_{2}\right)$ ou no bolso $\left(a_{1} b_{3}\right.$ e $\left.a_{2} b_{3}\right)$. Por fim, pode-se observar se o usuário está em movimento $\left(a_{1} b_{1} c_{1}, a_{1} b_{2} c_{1}, a_{1} b_{3} c_{1}\right.$, $a_{2} b_{2} c_{1}$ e $\left.a_{2} b_{3} c_{1}\right)$ ou parado $\left(a_{1} b_{1} c_{2}, a_{1} b_{2} c_{2}, a_{1} b_{3} c_{2}, a_{2} b_{2} c_{2}\right.$ e $\left.a_{2} b_{3} c_{2}\right)$.

Para adquirir a base de dados, utilizou-se um celular convencional equipado com um acelerômetro que mede as forças aplicadas ao dispositivo em um dado instante de tempo. A partir dessas forças, é possível extrair a direção e o sentido do movimento do aparelho através de sua aceleração instantânea. O valor da aceleração $\boldsymbol{a}$ é expresso como um vetor que apresenta as componentes, $\boldsymbol{a}_{x_{1}}, \boldsymbol{a}_{x_{2}}$, 


\begin{tabular}{|c|c|c|c|c|c|c|}
\hline Trabalho & Aplicação & Classes & Sensores & Classificador (es) & Tamanho da Janela & Taxa de Acerto \\
\hline Anjum and Ilyas (2013) & Rastreamento de atividades físicas. & $\begin{array}{l}\text { Inativo, caminhando, correndo, su- } \\
\text { bindo escadas, descendo escadas, diri- } \\
\text { gindo e andando de bicicleta. }\end{array}$ & Acelerômetro, giroscópio e GPS & $\begin{array}{l}\text { Arvore de Decisão, K-Nearest Neigh- } \\
\text { bor, Naive Bayes e SVM }\end{array}$ & 5 segundos & $94,4 \%$ \\
\hline Kwapisz et al. (2011) & Rastreamento de atividades físicas. & $\begin{array}{l}\text { Caminhando, correndo, subindo esca- } \\
\text { das,descendo escadas, ficando sentado } \\
\text { e ficando parado em pé. }\end{array}$ & Acelerômetro & $\begin{array}{l}\text { Arvore de Decisão J48, Regressão Lo- } \\
\text { gística e Redes Neurais Artificiais Mul- } \\
\text { ticamada }\end{array}$ & 10 segundos & $91,7 \%$ \\
\hline Aguiar et al. (2014) & Rastreamento de atividades físicas. & $\begin{array}{l}\text { Caminhando, correndo, ficando parado } \\
\mathrm{em} \text { pé, ficando sentado, ficando dei- } \\
\text { tado e atividades não listadas (trata- } \\
\text { das como "fora do escopo"). }\end{array}$ & Acelerômetro & Arvore de Decisão & 5 segundos & $99,5 \%$ \\
\hline Widhalm et al. (2012) & $\begin{array}{l}\text { Detecção de modo de transporte para } \\
\text { planejamento de cidades. }\end{array}$ & $\begin{array}{l}\text { Ónibus, carro, bicicleta, bonde, trem, } \\
\text { metrô, caminhada e moto. }\end{array}$ & Acelerômetro, GPS e rede do celular & Modelo Oculto de Markov Discreto & Variável de acordo com a velocidade & $75,88 \%$ \\
\hline Cardoso et al. (2016) & $\begin{array}{l}\text { Deteccão de modo de transporte para } \\
\text { o cuidado de idosos. }\end{array}$ & $\begin{array}{l}\text { Inativo, caminhando, ônibus, carro, } \\
\text { trem e metrô. }\end{array}$ & Acelerômetro e Wi-Fi & $\begin{array}{l}\text { Árvore de Decisão J48, Naive Bayes e } \\
\text { SMO }\end{array}$ & 5 segundos & Acima de $90 \%$ \\
\hline Mejia et al. (2016) & $\begin{array}{l}\text { Cálculo do risco de acidentes com ido- } \\
\text { sos. }\end{array}$ & $\begin{array}{l}\text { Estar em casa, ir ao centro e ir ao } \\
\text { mercado. }\end{array}$ & GPS & Modelo Probabilistico Próprio & & \\
\hline Figueira et al. (2016) & $\begin{array}{l}\text { Rastreamento de atividades físicas } \\
\text { para fisioterapia em idosos. }\end{array}$ & $\begin{array}{l}\text { Caminhando, correndo, subindo esca- } \\
\text { das, descendo escadas, ficando parado } \\
\text { em pé e ficando sentado. }\end{array}$ & Acelerômetro e barômetro & Árvore de Decisão & 5 segundos & $94,5 \pm 6,8 \%$ \\
\hline
\end{tabular}

Tabela 1. Resumo das abordagens e resultados obtidos em trabalhos correlatos.

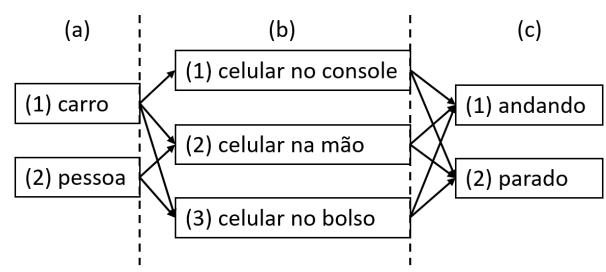

Figura 1. Dez situações, $\tau$, identificadas pela rede neural artificial. Alguns exemplos são: $a_{1} b_{1} c_{1}$ (carro, celular no console e andando), $a_{1} b_{1} c_{2}$ (carro, celular no console e parado), $a_{1} b_{2} c_{1}$ (carro, celular na mão e andando) etc.

$\boldsymbol{a}_{x_{3}}$, nos eixos $x_{1}, x_{2}$ e $x_{3}$, em unidades gravitacionais $\left(g=9.780327 \mathrm{~m} / \mathrm{s}^{2}\right)$. Para efeito de esclarecimento, a Figura 2 apresenta o sistema de coordenadas adotado pelo sensor, sendo $\hat{i}, \hat{j}, \hat{k}$ os vetores unitários dos eixos $x_{1}, x_{2}$ e $x_{3}$, respectivamente. Em outras palavras, a aceleração é dada por $\boldsymbol{a}=a_{x_{1}} \hat{i}+a_{x_{2}} \hat{j}+a_{x_{3}} \hat{k}$. A orientação da aceleração em relação ao dispositivo é definida de tal forma que $-1 g$ é aplicada ao dispositivo no eixo $x_{3}$ quando este encontrase no estado FaceUp ou, de maneira análoga, quando o vetor unitário $\hat{n}$ normal à tela do celular aponta na direção e sentido de $\hat{k}$.

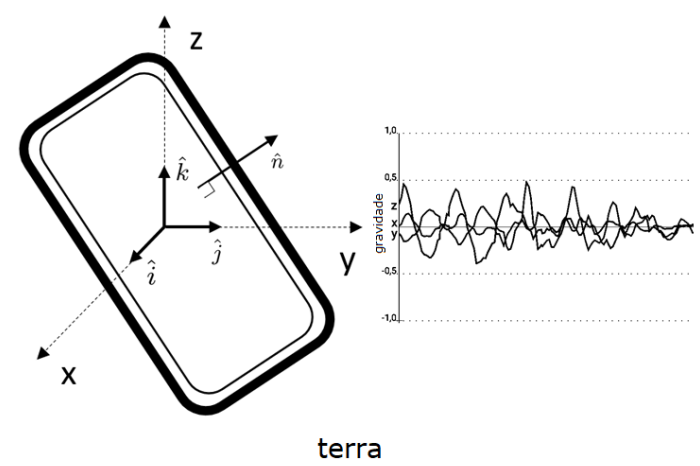

111111111111111111111111

Figura 2. Sistema de coordenadas de referência utilizado. Os vetores $\hat{i}, \hat{j}, \hat{k}$ são os vetores unitários dos eixos $x_{1}$, $x_{2}$ e $x_{3}$, respectivamente, e que $\hat{n}$ é o vetor unitário normal à tela do telefone celular.

Para cada uma das 10 possíveis situações apresentadas, 10 gravações de um minuto cada foram armazenadas localmente no celular. Posteriormente, cada gravação foi divida em 6 partes de 10 segundos. Aqui, cada uma dessas partes de 10 segundos é definida como uma amostra. É importante dizer que os sinais foram normalizados pelo valor da aceleração da gravidade e, portanto, variam entre -1 e 1 .

Por fim, a quantidade de amostras que compõem a base de dados é de 10 gravacoes $\times 6$ partes $\times 10$ classes 0 que perfaz um total de 600 amostras, das quais 300 são utilizadas para treinar e 300 são utilizadas para testar o classificador proposto sem que haja interseção entre o conjunto de treinamento e o conjunto de teste.

Uma vez gerada a base de dados, um vetor $\mathbf{X}$ de características é montado para cada uma das 600 amostras. Esse vetor é composto de 6 elementos conforme a Eq. 1 , onde $\overline{a_{x_{1}}}, \overline{a_{x_{2}}}, \overline{a_{x_{3}}}$ representam as médias aritméticas das amostras nos eixos $x_{1}, x_{2}$ e $x_{3}$; e $\sigma_{x_{1}}^{2}, \sigma_{x_{2}}^{2}$ e $\sigma^{2}{ }_{x_{3}}$ representam suas variâncias.

Por exemplo, o $i$-ésimo elemento $\left(\mathbf{X}_{i}, \tau_{i}\right)$ do conjunto de dados é classificado corretamente por $\tilde{\tau}_{i}=h\left(\mathbf{X}_{i}\right)$ se, e somente se, $\tilde{\tau}_{i}=\tau_{i}$, onde $\tau_{i}$ é a classe real, $\tilde{\tau}_{i}$ é a classe prevista, $\mathbf{X}_{i}$ é o vetor de entrada e $h(\cdot)$ é o sistema de classificação.

$$
\mathbf{X}=\left[\begin{array}{llllll}
\overline{a_{x_{1}}} & \overline{a_{x_{2}}} & \overline{a_{x_{3}}} & \sigma_{x_{1}}^{2} & \sigma_{x_{2}}^{2} & \sigma_{x_{3}}^{2}
\end{array}\right]^{t}
$$

Em nossos experimentos, um perceptron multicamada com 4 camadas é usado como o classificador. Para alcançar os pesos e vieses ótimos para as duas camadas ocultas, a seguinte estratégia de treinamento é usada: a quantidade de neurônios em cada camada é aumentada de 2 em 2 , começando em 10 e indo até 50 , ou seja, a primeira camada começa com um número fixo de 10 neurônios, enquanto a segunda camada tem seus neurônios aumentados de $2 \mathrm{em}$ 2 de 10 até 50 . Uma vez que a segunda camada atinge 50 neurônios, a primeira camada é alterada de forma a possuir 12 neurônios e a segunda varia novamente de 10 a 50. O processo de treinamento é repetido até que ambas as camadas possuam 50 neurônios. Cada saída passa por uma etapa de pós-processamento conhecida como winner-takes-all. A rede escolhida é aquela que apresenta o melhor desempenho utilizando o algoritmo de treinamento Levenberg-Marquardt. O experimento é composto por dois cenários diferentes, conforme apresentado abaixo.

\subsection{Cenário 1 - Uma entre 10 Possiveis Classes}

Nesse cenário, pretende-se identificar uma dentre as 10 possíveis classes apresentadas na Figura 1. Para tanto, utiliza-se uma rede neural artificial cuja arquitetura conta com uma camada de entrada com 6 neurônios, as duas camadas ocultas com 14 e 24 , respectivamente, e uma 
camada de saída com 10 neurônios. Exceto na camada de entrada, todos os neurônios utilizam a função de transferência tangente hiperbólica. Na entrada, apresenta-se um vetor conforme a Eq. 1 e a saída esperada (target) é um vetor $\Phi$ que identifica a classe de interesse conforme apresentado na Tabela 2. É possível observar que cada instância $\Phi_{n}$ corresponde a uma classe $\tau_{n}, n=1 . .10$.

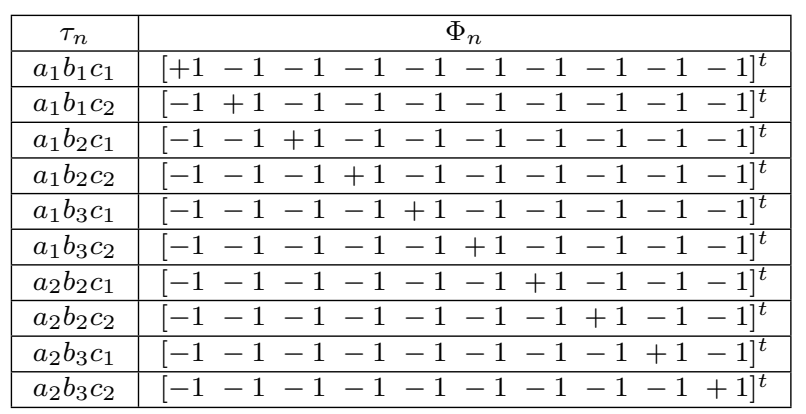

Tabela 2. Relação entre os vetores de saída $\Phi_{n}$ e os modos de transporte $\tau_{n}$.

\subsection{Cenário 2 - Carro X Não Carro}

Para diversas aplicações, é possível que se queira saber se o usuário do telefone encontra-se dentro de um veículo ou não. Este cenário tem especial relevância, pois indica uma situação de iminente risco. Mesmo que o usuário tenha colocado o celular no console, ele pode, a qualquer momento, tentar fazer uso do aparelho enquanto dirige. Neste cenário, um indicativo preventivo de risco pode ser disparado. As amostras são divididas em apenas duas classes: carro (união de todas as classes iniciadas com $a_{1}$ ) e $\operatorname{carro}^{c}$ (união de todas as classes iniciadas com $a_{2}$ ). Neste cenário, a camada de entrada possui 6 neurônios, as duas camadas ocultas 10 e 26 , respectivamente, e a camada de saída 1 neurônio. Assim como no cenário anterior, exceto pela camada de entrada, todas possuem a função de transferência tangente hiperbólica. Na entrada, é apresentado um vetor coluna conforme Eq. 1 e a saída esperada (target) é um número real $\Phi$ conforme apresentado na Eq. 2 .

$$
\Phi= \begin{cases}+1, & \text { if carro } \\ -1, & \text { if } \text { carro }^{c}\end{cases}
$$

Na próxima seção, são apresentados os resultados.

\section{RESULTADOS}

Os resultados foram sintetizados na forma de matrizes de confusão. Como no Cenário 1 o número de classes a serem preditas é 10 , a matriz de confusão correspondente apresentaria dimensão $10 \times 10$. Sendo assim, para facilitar a organização dos resultados, utilizaram-se duas matrizes $5 \times 5$. É possível observar, na Tabela 3 , que apenas 3 das 10 classes foram preditas com menos de $95 \%$ de acerto e 5 classes foram preditas com $100 \%$ de acerto. A classe com o menor percentual de acerto $(84.4 \%)$ é a em que o usuário está parado segurando o telefone $\left(a_{2} b_{2} c_{2}\right)$. Em maior parte, essa classe é confundida com a situação em que o usuário está andando com o celular na mão. Com relação ao Cenário 2, nota-se que a tarefa de distinguir o meio de transporte com o qual o usuário está se locomovendo (a pé ou de carro) foi resolvida de forma satisfatória, com $100 \%$ de acerto, conforme mostrado na Tabela 4.

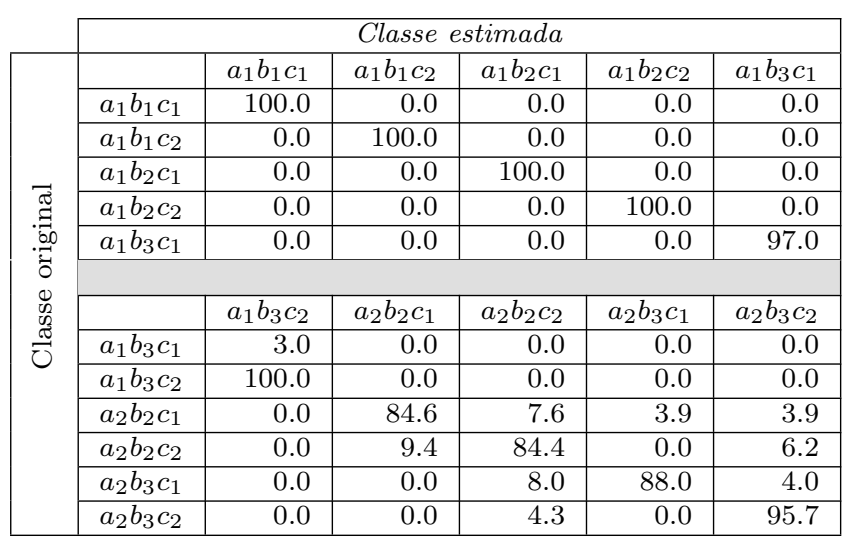

Tabela 3. Matriz de confusão para o Cenário 1.

\begin{tabular}{|r|r|r|}
\hline & $a_{1}$ & $a_{2}$ \\
\hline$a_{1}$ & 100,0 & 0,0 \\
\hline$a_{2}$ & 0,0 & 100,0 \\
\hline
\end{tabular}

Tabela 4. Matriz de confusão para o cenário 2.

\section{CONCLUSÃO}

Descobrir qual o comportamento de um indivíduo a partir dos dados capturados e armazenados por seu telefone celular é sem dúvida um tema atual e de interesse, principalmente quando se tem em mente que a utilização inadequada de tais dispositivos, como em situações de trânsito, pode causar acidentes fatais. Este artigo apresentou um método simples, mas eficiente, para detectar determinados comportamentos padrão utilizando sinais oriundos do acelerômetro nativo contido em telefones celulares convencionais. Resultados globais de $95 \%$ e $100 \%$ de acerto, para os Cenários 1 e 2, respectivamente, foram obtidos utilizando-se um classificador baseado em redes neurais artificiais que toma como entrada um vetor de características cuja computação requer reduzido poder computacional. A classe que apresenta o maior risco de acidente é aquela em que o carro está em movimento enquanto o usuário está com o celular na mão $\left(a_{1} b_{2} c_{1}\right)$. É possível observar que o classificador foi capaz de identificar esta situação em $100 \%$ dos casos. Percebe-se também que a rede neural artificial proposta no Cenário 2 (100\% de acerto) pode ser empregada para refinar a classificação das situações em que o usuário não está dentro do carro (classes com maior percentual de erro no Cenário 1). Tal rede pode ser utilizada como a primeira etapa de um classificador hierárquico. Dessa forma, fica demonstrado o potencial da abordagem aqui proposta. Trabalhos futuros pretendem incluir novas classes de interesse e, como consequência, a proposta de novas e mais eficientes características distintivas. Além disso, pretende-se construir uma base de dados maior e disponibilizá-la para a comunidade científica com o objetivo de aprimorar os métodos de classificação. 


\section{REFERÊNCIAS}

Aguiar, B., Silva, J., Rocha, T., Carneiro, S., and Sousa, I. (2014). Monitoring physical activity and energy expenditure with smartphones. In IEEE-EMBS International Conference on Biomedical and Health Informatics (BHI), 664-667. doi:10.1109/BHI.2014.6864451.

Anjum, A. and Ilyas, M.U. (2013). Activity recognition using smartphone sensors. In 2013 IEEE 10th Consumer Communications and Networking Conference (CCNC), 914-919. doi:10.1109/CCNC.2013.6488584.

Ayers, J.W., Leas, E.C., Dredze, M., Allem, J.P., Grabowski, J.G., and Hill, J. (2016). Pokémon go - a new distraction for drivers and pedestrians. JAMA Internal Medicine, 176, 1865-1866.

Cardoso, N., Madureira, J., and Pereira, N. (2016). Smartphone-based transport mode detection for elderly care. In 2016 IEEE 18th International Conference on e-Health Networking, Applications and Services (Healthcom), 1-6. doi:10.1109/HealthCom.2016.7749465.

Figueira, C., Matias, R., and Gamboa, H. (2016). Body location independent activity monitoring. In Proceedings of the 9th International Joint Conference on Biomedical Engineering Systems and Technologies - Volume 4: BIOSIGNALS, (BIOSTEC 2016), 190-197. doi:10.5220/ 0005699601900197.

Kwapisz, J.R., Weiss, G.M., and Moore, S.A. (2011). Activity recognition using cell phone accelerometers. SIGKDD Explor. Newsl., 12(2), 74-82. doi:10. 1145/1964897.1964918. URL http://doi .acm.org/10. 1145/1964897. 1964918.

Mejia, M.E.L., Montalvo, J.R.G., Mena, F.M., and Ramirez, F.A.M. (2016). Intelligent platform for non intrusive assistance of elderly people. IEEE Latin America Transactions, 14(5), 2433-2439. doi:10.1109/TLA.2016. 7530442.

Sahyoun, A., Chehab, K., Al-Madani, O., Aloul, F., and Sagahyroon, A. (2016). Parknosis: Diagnosing parkinson's disease using mobile phones. In 2016 IEEE 18th International Conference on e-Health Networking, Applications and Services (Healthcom), 1-6. doi:10.1109/ HealthCom.2016.7749491.

Shapsough, S., Hesham, A., Elkhorazaty, Y., Zualkernan, I.A., and Aloul, F. (2016). Emotion recognition using mobile phones. In 2016 IEEE 18th International Conference on e-Health Networking, Applications and Services (Healthcom), 1-6. doi:10.1109/HealthCom.2016. 7749470 .

Sigloch, W. (2016). Pedestrians crossing streets: Distraction by smartphone poses risks. Technical report, DEKRA, Handwerkstraße 15, D-70565 Stuttgart. URL www. dekra.com/en/press.

Widhalm, P., Nitsche, P., and Brändie, N. (2012). Transport mode detection with realistic smartphone sensor data. In Proceedings of the 21st International Conference on Pattern Recognition (ICPR2012), 573-576. 